\title{
Reliable Detection of a Variance Increase in a Critical Process Variable
}

\author{
Mika Pylvänäinen Toni Liedes \\ Mechatronics and Machine Diagnostics Research Unit, University of Oulu, Finland, \\ \{mika.pylvanainen, toni. liedes\} @oulu.fi
}

\begin{abstract}
Industrial process failures can be often seen as a variance increase in a measured process variable. The objective of this research was to investigate if stochastic Autoregressive Moving Average, abbreviated as ARMA, and Generalized Autoregressive Conditionally Heteroscedastic, abbreviated as GARCH, time series modelling are feasible methods for the reliable detection of gradually increasing variance in the process variable. A case study was conducted for the reliable detection of increased pressure variance that indicates a harmful air leakage in a vacuum chamber in a paper machine. Variance in the chamber pressure was artificially gradually increased, a combined ARMA+GARCH time series model was fitted to it and the variance vector was determined. An abnormally high variance was detected from the variance vector using a specified detection limit and detection sensitivity. According to the simulation results, by controlling the variance vector extracted from the combined ARMA+GARCH time series model, a very slight variance increase in the process variable can be detected more reliably than detecting it from the moving variance vector computed directly from the process variable.
\end{abstract}

Keywords: condition monitoring, time series analysis, $S P C$

\section{Introduction}

The variance increase of the critical process variable is often an early sign of an abnormal process behavior (Jandhyala et al., 2002; Du et al., 2010). It can be also a sign of a phenomenon that may escalate to an unplanned process shutdown or severe damage in a critical process component (Rzadkowski et al., 2016). This can cause a significant loss in production capacity due to an unplanned maintenance or the long delivery time of a spare part. Therefore, it is crucial to reliably detect an abnormal variance increase in real time process condition monitoring. This enables a transition from reactive maintenance to condition-based maintenance.

In the paper manufacturing process, there are several critical process variables whose variance increase is a sign of incipient failure.

One typical example is increased gauge pressure variance, later called pressure variance, in a certain vacuum chamber in a paper machine. In a stable and faultless paper manufacturing process, the mean of the vacuum chamber pressure is constant while its variance inherently fluctuates within a certain range.

According to process specialists, air leakage in the vacuum chamber gradually increases variance in the chamber pressure while the pressure mean remains constant. When air leakage increases, also the pressure variance increases until the pressure value exceeds its tolerance limit. This triggers an operation sequence of a certain mechanical function, the sound of which is subjectively interpreted as a sign of air leakage. Instead of the subjective interpretation of a sound in a noisy paper machine environment, an incipient air leakage in the vacuum chamber could be reliably identified if an abnormal variance increase is detected from the chamber pressure. This improves product quality, enables preventive actions and helps avoid costly process breakdowns.

Numerous time series analysis methods are used in economics to model and predict econometric phenomena. Two commonly used analysis methods in economics are Autoregressive Moving Average, abbreviated as ARMA, and Generalized Autoregressive Conditionally Heteroscedastic, abbreviated as GARCH, time series modelling. Although several papers (Wang et al., 2002; Tao et al., 2010) have been published on the separate use of these methods in condition monitoring, fewer studies (Pham et al., 2010; Caesarendra et al., 2011) have been published on the joint use of the ARMA and GARCH time series models in this field. These studies prove that the combined ARMA +GARCH time series model provides a versatile and powerful toolkit for condition monitoring.

The objective of this research was to investigate if the combined ARMA+GARCH time series modelling is a feasible method for the reliable detection of increased variance in a critical process variable. Data analysis was done by $\mathrm{R}$ software.

A case study was conducted for the detection of increased pressure variance in a vacuum chamber in the paper machine. At the beginning of the study, chamber pressure data were recorded at $8 \mathrm{~s}$ sampling intervals in a $140 \mathrm{~h}$ period. During that time, there was no air leakage in the vacuum chamber. 
Then the ARMA model was fitted to the data. Autocorrelation of the squared residuals of the fitted ARMA model indicated that they still contain information so the residuals were further modelled using the GARCH model. The squared residuals of the fitted GARCH model were no longer autocorrelated. Thus all the information included in the chamber pressure data was decomposed to the coefficients of the fitted ARMA +GARCH model. The model defines a typical behavior for vacuum pressure when air leakage does not occur.

Due to missing air leakages in the vacuum chamber during the study, the vacuum pressure vector including the air leakage effect was artificially created by first simulating the pressure vector using the combined ARMA+GARCH model with coefficients representing the process without air leakage. Then a vector with mean zero and slightly increasing variance was summed to the simulated pressure vector to mimic air leakage. The sum vector represents the chamber pressure in an incipient air leakage condition. The goal in the next steps of the study is to detect the systematic variance increase in the chamber pressure with minimum delay.

An artificially created pressure vector was modelled by means of the combined ARMA+GARCH model using the same number of coefficients as in the stable process model. The variance vector extracted from the aforementioned model illustrates the variance behavior of the pressure vector. It is challenging to detect variance increase caused by air leakage due to an inherent variance fluctuation even when the air leakage is not present. An alarm for an abnormally high variance in the vacuum pressure is triggered when the variance vector values exceed a quantile that is defined using an adjustable detection limit, abbreviated as $p_{k}$. The quantile divides an empirical distribution of past variance values so that $p_{k}$ proportion of variance values are smaller than the quantile. To avoid false alarms, adjustable detection sensitivity, abbreviated as $s$, is used to specify the minimum number of consecutive values of the variance vector above the quantile before the alarm is triggered. Once the alarm is triggered, a delay, i.e. number of pressure observations from the start of the leakage until its detection, is recorded.

The sequence described above was simulated $27 \cdot 10^{3}$ rounds. According to the simulation results, it is possible to detect an almost invisible variance increase in the vacuum chamber pressure more reliably than detecting it from the moving variance vector computed from the process variable.

\section{Time Series Models and Detection Parameters}

In this chapter the Autoregressive model, the Moving Average model and their combination called Autoregressive Moving Average model are examined. Then the Generalized Autoregressive Conditionally
Heteroscedastic model is introduced and description is given how the autocorrelated time series vector is decomposed by the combined use the Autoregressive Moving Average model and the Generalized Autoregressive Conditionally Heteroscedastic model. Finally, a detection limit and detection sensitivity of abnormally high variance detection in the time series vector is examined.

\subsection{Autoregressive Moving Average Model}

The ARMA model is the most popular class of linear time series models. ARMA models are commonly used to model linear dynamic structures and, to describe a linear relationship among lagged variables, i.e. when the variable is autocorrelated. The ARMA model consists of two submodels, the Autoregressive model, abbreviated as AR, and the Moving Average model, abbreviated as MA.

The Autoregressive model of order $p$, abbreviated as $\operatorname{AR}(p)$, is of the form

$$
x_{t}=\phi_{1} x_{t-1}+\phi_{2} x_{t-2}+\ldots+\phi_{p} x_{t-p}+w_{t},
$$

where $x_{t}$ is a stationary time series with mean zero; $\phi_{1}, \phi_{2}, \ldots, \phi_{p}$ are constants $\left(\phi_{p} \neq 0\right)$ and $w_{t}$ is a Gaussian white noise with mean zero and variance $\sigma_{w}{ }^{2}$. The $\operatorname{AR}(p)$ model assumes that the current value of time series $x_{t}$ is defined as a sum of the linear combination of $p$ past time series values and the white noise $w_{t}$ (Shumway et al., 2011; Box et al., 1976).

The Moving Average model of order $q$, abbreviated $\operatorname{MA}(q)$ is of the form

$$
x_{t}=w_{t}+\theta_{1} w_{t-1}+\theta_{2} w_{t-2}+\ldots+\theta_{p} w_{t-q},
$$

where $x_{t}$ is a stationary time series with mean zero; $\theta_{1}, \theta_{2}, \ldots, \theta_{q}\left(\theta_{q} \neq 0\right)$ are the constant parameters and $w_{t}$ is the Gaussian white noise with mean zero and variance $\sigma_{w}{ }^{2}$. The $\operatorname{MA}(q)$ model assumes that the current value of time series $x_{t}$ is defined as the linear combination of the current and $q$ previous values of the white noise $w_{t}$ (Shumway et al., 2011; Box et al., 1976).

When the Autoregressive model $\operatorname{AR}(p)$ and the Moving Average model $\operatorname{MA}(q)$ are combined, the Autoregressive Moving Average model of order $p$ and $q$, abbreviated as $\operatorname{ARMA}(p, q)$ is defined. $\operatorname{ARMA}(p, q)$ is of the form

$$
x_{t}=\phi_{1} x_{t-1}+\ldots+\phi_{p} x_{t-p}+w_{t}+\theta_{1} w_{t-1}+\ldots+\theta_{p} w_{t-q} .
$$

The ARMA model assumes that the variance of the time series $x_{t}$ is constant. This is not always the case. Thus varying variance cannot be modelled using the ARMA model and therefore its residuals are not Gaussian white noise, i.e. they still contain information (Shumway et al., 2011; Box et al., 1976). 


\subsection{Generalized Autoregressive Conditionally Heteroscedastic Model}

The Generalized Autoregressive Conditionally Heteroscedastic model, abbreviated as GARCH, is frequently used in economics and finance to model the varying variance of the time series $x_{t}$, i.e. when the variance of the time series is autocorrelated. The GARCH modelling is used to model such information from time series $x_{t}$ that is not possible with the ARMA modelling. For example, autocorrelated squared residuals of the ARMA model indicate that they still contain information that cannot be modelled by means of the ARMA model. Then the GARCH is used to model the information included in the residuals of the ARMA model.

The Generalized Autoregressive Conditionally Heteroscedastic model of order $m$ and $r$, abbreviated as $\operatorname{GARCH}(m, r)$, is of the form

$$
\begin{gathered}
w_{t}=\sigma_{t} \varepsilon_{t} \\
\sigma_{t}^{2}=\alpha_{0}+\sum_{j=1}^{m} \alpha_{j} w_{t-j}^{2}+\sum_{j=1}^{r} \beta_{j} \sigma_{t-j}^{2}
\end{gathered}
$$

where $w_{t}$ is noise, i.e. the residual of the ARMA model with mean zero, $\sigma_{t}^{2}$ is the variance of the noise, $\varepsilon_{t}$ is standard Gaussian white noise $\varepsilon_{t} \sim \operatorname{iidN}(0,1) ; \alpha_{0}$, $\alpha_{1}, \alpha_{2}, \ldots, \alpha_{m}, \beta_{1}, \beta_{2}, \ldots, \beta_{r}$ are constants $\left(\alpha_{0}>0, \alpha_{j} \geq 0, \beta_{j}\right.$ $\geq 0)$. The $\operatorname{GARCH}(m, r)$ model assumes that the variance $\sigma_{t}^{2}$ is defined as the sum of the constant $\alpha_{0}$, the linear combination of $m$ past values of a squared noise $w_{t}{ }^{2}$ and the linear combination of $r$ past values of variance $\sigma_{w}^{2}$ (Shumway et al., 2011; Cowpertwait et al., 2009; Bollerslev, 1986).

The flowchart in Fig. 1 illustrates the decomposition of the autocorrelated time series vector $x_{t}$ in the combined ARMA+GARCH time series model. As can be seen in the Fig. 1, data decomposition is performed consecutively, first in the fitted ARMA model and then in the fitted GARCH model. In the model fitting, model coefficients are determined so that the sum of the squared model estimate errors is minimized. This fitting approach is called the least squares method, abbreviated as LS method. The LS method is commonly used in model fitting and will also be used in this research.

The estimate error, i.e. residual, is the deviation between the data point and the corresponding value estimated using the model. In Fig. 1, estimate errors, i.e. residuals of the fitted ARMA model and the fitted GARCH model are represented by the residual vector $w_{t}$ and the standard Gaussian white noise vector $\varepsilon_{t}$.

The plots in Fig. 2, sections (a-d), are based on the simulated time series vector $x_{t}$ with $2 \cdot 10^{3}$ observations. They illustrate the behavior of the time series vector $x_{t}$, residual vector $w_{t}$, the variance vector $\sigma_{t}^{2}$ and the standard Gaussian white noise vector $\varepsilon_{t}$.
When comparing the plots in sections $(a-b, d)$, it can be clearly seen that the information content of these vectors decreases along the decomposition steps.

In the first decomposition, the ARMA model is fitted to the time series vector $x_{t}$. As illustrated in Fig. 1, the fitted model explains the AR effect by decomposing the data to coefficients $\phi_{1}, \phi_{2}, \ldots, \phi_{p}(1)$.

The MA effect is explained by decomposing the data to coefficients $\theta_{1}, \theta_{2}, \ldots, \theta_{q}(2)$. Unlike the time series vector $x_{t}$, the residual vector $w_{t}$ is not autocorrelated because the autocorrelation effect has already been decomposed to the AR and MA coefficients. However, if a squared residual vector i.e. $w_{t}^{2}$ is autocorrelated, the residual vector $w_{t}$ contains information on varying variance of the time series vector $x_{t}$, which could not be explained using the fitted ARMA model.

The second decomposition is performed for the residual vector $w_{t}$ in order to explain its varying variance information. Thus the GARCH model is fitted to the residual vector $w_{t}$. The fitted GARCH model decomposes information on varying variance to the coefficients $\alpha_{0}, \alpha_{1}, \alpha_{2}, \ldots, \alpha_{m}, \beta_{1}, \beta_{2}, \ldots, \beta_{r}$. Consequently, the standard Gaussian white noise vector $\varepsilon_{t}$ contains no information as can be seen in Fig. 2, section (d), because $\varepsilon_{t} \sim$ iidN $(0,1)$. Therefore the values of the vector $\varepsilon_{t}$ are independent and identically and normally distributed with mean zero and variance one. In addition, the standard Gaussian white noise vector $\varepsilon_{t}$ and its square are not autocorrelated because all the remaining information in the residual vector $w_{t}$ is decomposed to the coefficients of the fitted GARCH model. The variance vector $\sigma_{t}^{2}$ in Fig. 2, section (c), is determined based on these coefficients (5).

As mentioned before, the ARMA model cannot explain the varying variance included in the time series vector $x_{t}$ (Shumway et al., 2011; Box et al., 1976). Consequently, the residual vector $w_{t}$ contains unexplained varying variance information so it makes variance behavior of the time series vector $x_{t}$ clearly visible. Therefore, the variance vector $\sigma_{t}^{2}$ in Fig. 2, section (c), illustrates the variance behavior of the time series vector $x_{t}$ although it is determined on the basis of the residual vector $w_{t}$.

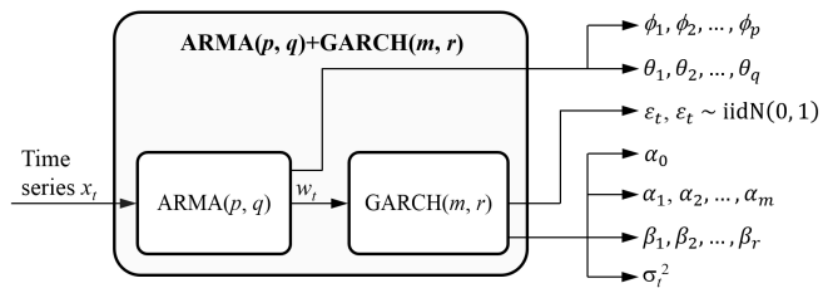

Figure 1. Decomposition of time series vector $x_{t}$ to residual vector $w_{t}$, variance vector $\sigma_{t}^{2}$, standard Gaussian white noise vector $\varepsilon_{t}$ and coefficients $\phi_{1}, \phi_{2}, \ldots, \phi_{p}, \theta_{1}, \theta_{2}, \ldots, \theta_{q}$, $\alpha_{0}, \alpha_{1}, \alpha_{2}, \ldots, \alpha_{m}, \beta_{1}, \beta_{2}, \ldots, \beta_{r}$. 


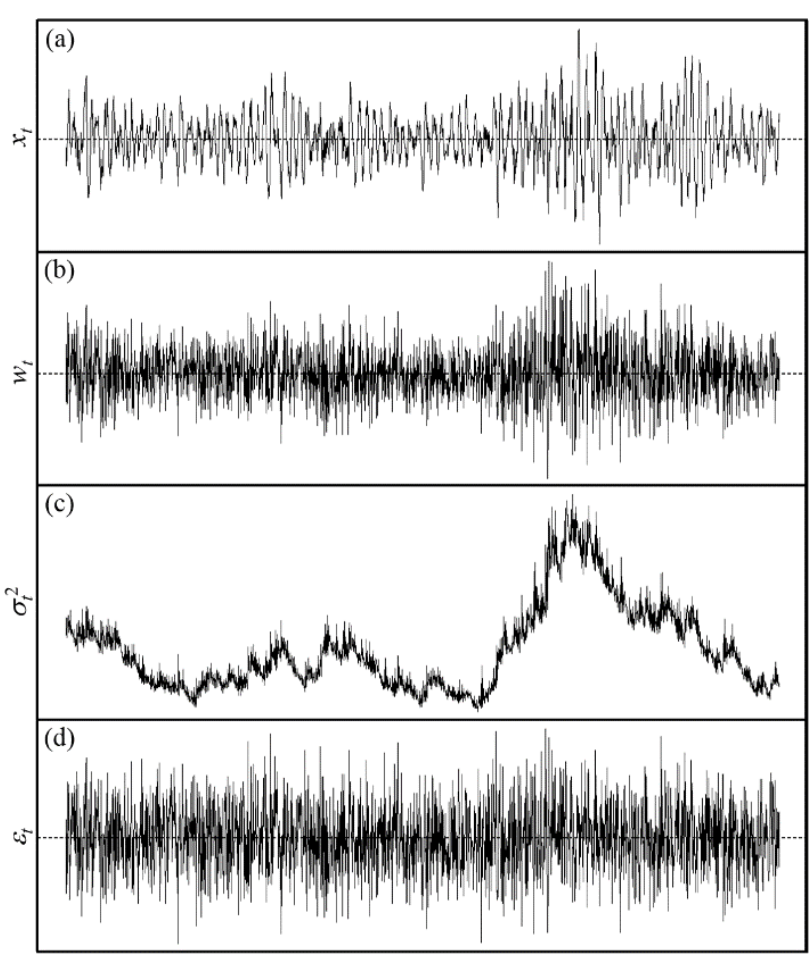

Figure 2. (a) Time series vector $x_{t}$, (b) residual vector $w_{t}$, (c) variance vector $\sigma_{t}^{2}$ and (d) standard Gaussian white noise vector $\varepsilon_{t}$.

\subsection{Detection Limit and Detection Sensitivity}

An incipient process failure causing abnormally high values in the variance vector $\sigma_{t}^{2}$ is challenging to identify, because the variance also fluctuates inherently in the stable process condition. Based on simulations, the empirical distributions of the variance vector values were not normal. Therefore, the Statistical Process Control charts, abbreviated SPC charts, for identifying abnormally high variance values could not be used due to their underlying assumptions of normality (Montgomery, 2009).

In case the empirical distribution of variance vector values is not normal, abnormally high variance values can be detected when they exceed a quantile of the empirical distribution (Montgomery, 2009). The empirical distribution of the variance vector $\sigma_{t}^{2}$ is defined based on the process period that is known to be stable. The quantile is set by an adjustable detection limit, abbreviated as $p_{k}$. The quantile, later called $p_{k}$ quantile, divides an empirical distribution so that $p_{k}$ proportion of the variance values is smaller than the $p_{k}$ quantile. $p_{k} \in[0,1]$. When the objective is to detect abnormally high variance values, the detection limit $p_{k}$ is set close to one.

Although the variance vector $\sigma_{t}^{2}$ values are at a reasonably low level, they may still have individual high peak values, i.e. outliers triggering a false alarm of an abnormally high variance. The variance vector may also have an ascending trend while an individual high peak triggers a premature alarm even though the general variance level is below the $p_{k}$ quantile.

The number of false alarms can be reduced using adjustable detection sensitivity, abbreviated as $s$, which specifies the minimum number of the consecutive values of the variance vector $\sigma_{t}^{2}$ above the $p_{k}$ quantile before the alarm is triggered.

\section{Case Study and Simulation}

This chapter introduces a case study conducted in the papermaking industry. Objective was to detect a problematic air leakage as early as possible in a paper machine. Steps of the combined ARMA+GARCH time series model use for an incipient air leakage detection are examined. Ability of the examined method to detect air leakage with minimum delay was tested by simulation.

\subsection{Background and the Process Data}

An industrial case study was conducted for a paper machine, where it is critical to reliably detect an incipient air leakage in a certain vacuum chamber. Based on experience, air leakage in the vacuum chamber gradually increases variance in the chamber pressure while its mean still remains constant. When air leakage increases, the pressure variance also increases until the pressure value exceeds its tolerance limit. Crossing the tolerance limit of the vacuum pressure triggers a sequence of certain mechanical functions in the process, causing an audible sound that is currently subjectively interpreted as a sign of air leakage.

Fig. 3 illustrates the behavior of the pressure in the vacuum chamber. The pressure data used in the case study were recorded at $8 \mathrm{~s}$ sampling intervals during a $140 \mathrm{~h}$ period. The pressure is stationary, i.e. it varies around the constant mean. Therefore, there is no need for differencing to make it compatible with the ARMA model (Shumway et al., 2011). Pressure variance slightly increases at $70 \mathrm{~h}$ of the time. Although the variance increase is detectable, it may be caused by an inherent fluctuation of the pressure variance in a stable and faultless process condition.

\subsection{Fitting of a Combined ARMA+GARCH Model}

In order to define a typical behavior of the chamber pressure in terms of the coefficients of the combined ARMA+GARCH model, the model is fitted to such a period of the pressure data that represents the stable and faultless process. According to an interview of the paper machine specialists, there were no air leakages observed during the data collection. Thus the pressure behavior including the variance increase at $70 \mathrm{~h}$ of the time, as shown in Fig. 3, is considered to be typical for the process. Therefore, the combined ARMA+GARCH model was fitted to the data of the whole $140 \mathrm{~h}$ period. 


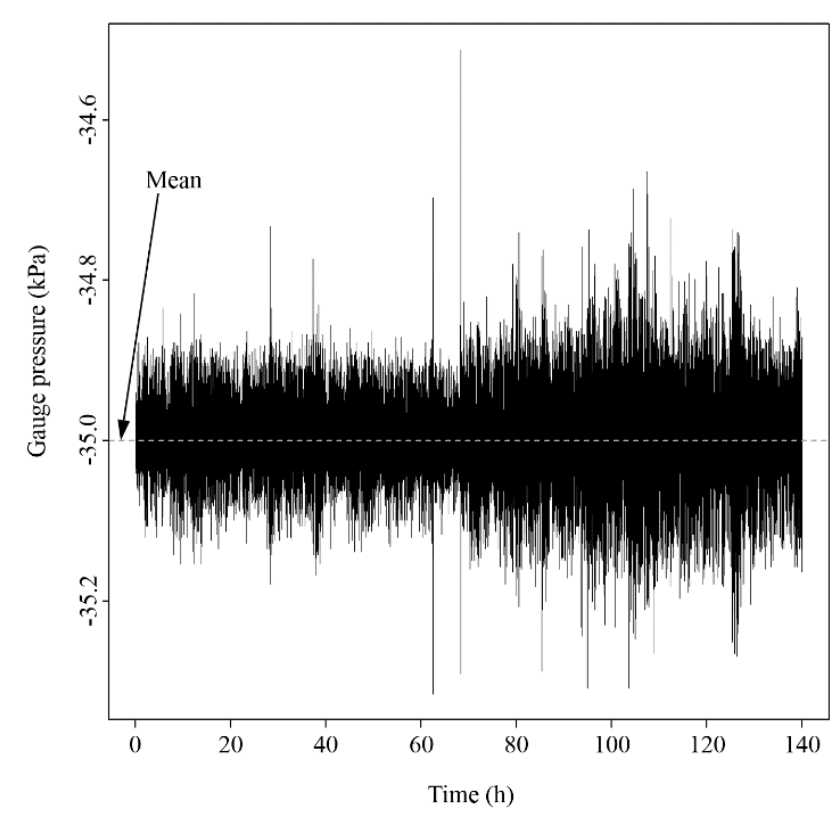

Figure 3. Gauge pressure of the vacuum chamber in the paper machine. Data were recorded at $8 \mathrm{~s}$ sampling intervals during a $140 \mathrm{~h}$ period.

The best fitting to the data was found when the combined ARMA $(4,4)+\operatorname{GARCH}(1,5)$ model was used. This means that the ARMA model orders are $p=4, q=$ 4 and the GARCH model orders are $m=1, r=5$.

Based on the definitions of the ARMA model (3) and GARCH model $(4,5)$, the combined $\operatorname{ARMA}(4,4)$ $+\mathrm{GARCH}(1,5)$ model is of the form

$$
\begin{aligned}
x_{t}= & -0.6790+2.1828 \cdot x_{t-1}-1.1515 \cdot x_{t-2} \\
& -0.3733 \cdot x_{t-3}+0.3226 \cdot x_{t-4}+\sigma_{t} \varepsilon_{t} \\
& -1.6268 \cdot \sigma_{t-1} \varepsilon_{t-1}+0.3339 \cdot \sigma_{t-2} \varepsilon_{t-2} \\
& +0.4576 \cdot \sigma_{t-3} \varepsilon_{t-3}-0.1537 \cdot \sigma_{t-4} \varepsilon_{t-4} \\
\sigma_{t}^{2}= & 0.000001+0.036782 \cdot\left(\sigma_{t-1} \varepsilon_{t-1}\right)^{2} \\
& +0.323369 \cdot \sigma_{t-1}^{2}+0.097586 \cdot \sigma^{2}{ }_{t-4} \\
& +0.541243 \cdot \sigma^{2}{ }_{t-5}
\end{aligned}
$$

The terms of the equation (7), which are related to $\sigma_{t-2}^{2}$ and $\sigma_{t-3}^{2}$, were omitted because their coefficients were not statistically significant, i.e. zero was included in their $95 \%$ confidence interval.

\subsection{Air Leakage Simulation}

Since no air leakage occurred in the vacuum chamber during the data collection, it had to be created artificially through simulation. Fig. 4, sections (a-c), illustrate the simulation steps from left to right in the same y-axis scale. At the beginning, in Fig. 4, section (a), the time series vector $x_{t}$ with $5 \cdot 10^{3}$ observations is simulated according to the combined $\operatorname{ARMA}(4,4)+\operatorname{GARCH}(1,5)$ model that was determined in the previous chapter. The simulated time series vector $x_{t}$ represents the typical behavior of vacuum chamber pressure in the stable and faultless process condition.

A disturbance vector with $5 \cdot 10^{3}$ observations is simulated to mimic an incipient and gradually increasing air leakage in the vacuum chamber. The first $2 \cdot 10^{3}$ observations are constant zero, due to the initial phase of the process that is not disturbed. Fig. 4, section (b), illustrates with a vertical dashed line how since the start of the leakage the variance of the normally distributed random vector linearly increases from zero to its maximum, which is set to low in order to make the disturbance challenging to detect.

The simulated vector in Fig. 4, section (a), and the disturbance vector in section (b) are summed. The sum vector, illustrated in section (c) mimics a pressure of the vacuum chamber in an incipient and gradually increasing air leakage condition that starts after $2 \cdot 10^{3}$ observations.

The sum vector in Fig. 4, section (c), represents the time series vector $x_{t}$ as in Fig. 2, section (a), and is decomposed in the fitted combined $\operatorname{ARMA}(4,4)$ $+\operatorname{GARCH}(1,5)$ model. In the decomposition, the variance vector $\sigma_{t}^{2}$ is determined (5) based on the coefficients of the fitted GARCH $(1,5)$ model. The steps described in this chapter are repeated in each simulation round.

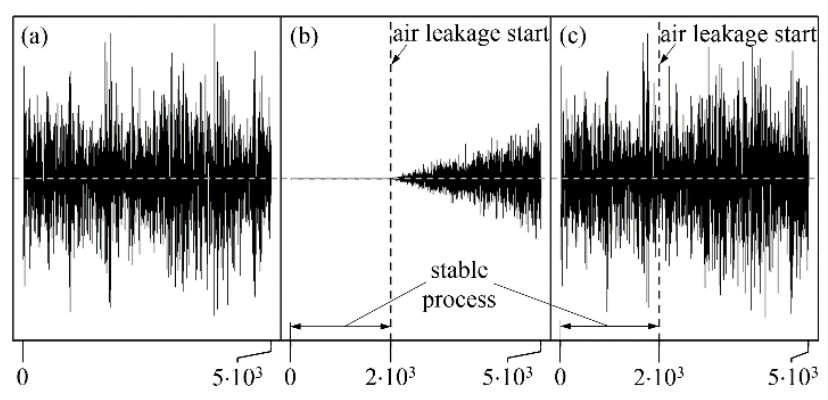

Figure 4. (a) Simulated pressure vector representing the stable and faultless process that follows the combined $\operatorname{ARMA}(4,4)+\mathrm{GARCH}(1,5)$ model. (b) Disturbance vector representing an increasing air leakage. (c) Sum vector that includes the simulated pressure vector and the disturbance vector.

\subsection{Air Leakage Detection}

Air leakage, i.e. an abnormally high variance in vacuum chamber pressure, should be detected from the variance vector $\sigma_{t}^{2}$ with as short a delay as possible after the start of the air leakage. Fig. 5 illustrates the behavior of the variance vector $\sigma_{t}^{2}$ in one simulation round. A vertical dashed line illustrates a time point when an incipient and gradually increasing air leakage starts, as in Fig. 4, section (b). Before the start of the air leakage, the variance vector $\sigma_{t}^{2}$ represents the stable process. That part of the vector is used for defining the detection threshold of an abnormally high variance. In the threshold setting, the $p_{k}$ quantile was defined by setting the detection limit $p_{k}=0.999$. 
Thus only $1 \%$ of the variance vector observations during the stable process are above the $p_{k}$ quantile. In case it occurs, it is considered a rare event and therefore an abnormally high variance observation.

In Fig. 5, after some delay since the start of the air leakage, variance vector $\sigma_{t}^{2}$ adopts an ascending trend until its first values cross the pk quantile. As can be seen, the first crossings of the $p_{k}$ quantile do not trigger an alarm of an abnormally high variance due to detection sensitivity $s$. As soon as $s$ pcs of the consecutive observations above the $p_{k}$ quantile are counted, an alarm for an abnormally high variance is triggered and a detection delay, abbreviated as $d_{i}$, of the simulation round $i$ is recorded. $d_{i}$ describes the delay counted from the start of the air leakage until its detection as the number of variance vector $\sigma_{t}^{2}$ observations.

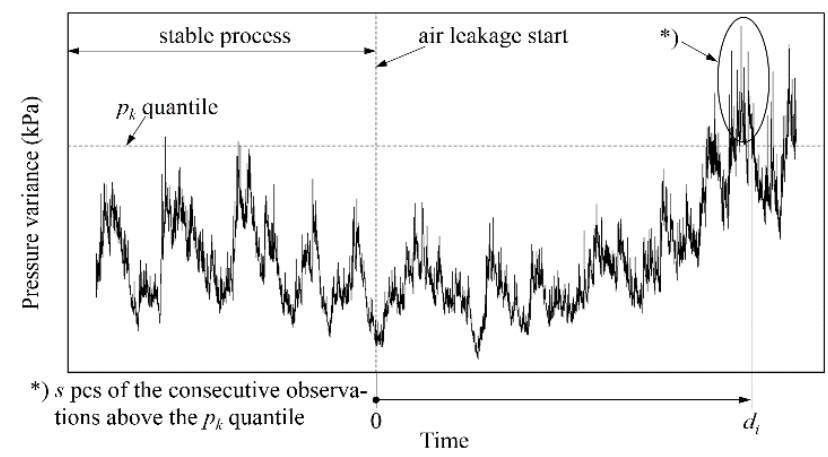

Figure 5. Vector of pressure variance in an air leakage simulation round $i, i=1,2, \ldots, N_{\text {sim. }}$. Air leakage starts at time point zero at vertical dashed line. Pressure variance $\sigma_{t}^{2}$ increases towards the right until the air leakage is detected in delay $d_{i}$, when $s$ pcs of the consecutive variance vector observations above the $p_{k}$ quantile are counted.

The detection delay $d_{i}$ is recorded in each simulation round $i, i=1,2, \ldots, N_{\text {sim }}$, In this study, the $N_{\text {sim }}=27 \cdot 10^{3}$. The detection delays of all the simulation rounds were summarized in an empirical cumulative probability curve illustrated in Fig 6, section (a). The cumulative probability curve refers the probability to detect an air leakage using the given delay.

Detection sensitivity $s$ has an effect onto the shape and location of the cumulative probability curve. The lower the $s$ is, the higher is the probability to detect an air leakage using the given delay, i.e. the detection becomes more sensitive. On the other hand, the lower the $s$ is, the greater is the type I error (Montgomery, 2009), i.e. the greater is the likelihood of a false air leakage alarm in the stable process phase when there is actually no air leakage. Based on the simulation data, the detection sensitivity was set to $s=60$ in order to limit the maximum of the type I error rate to $10 \%$. If the type I error rate needs to be lowered, it reduces the sensitivity of the air leakage detection. Thus the compromise between these two depends on the cost of making type I error and the cost of not being able to detect air leakage on time.
Fig. 6, section (a), illustrates that when controlling the variance vector $\sigma_{t}^{2}$ of the combined $\operatorname{ARMA}(4,4)$ $+\operatorname{GARCH}(1,5)$ model, half of the air leakages were detected with a delay of 2214 observations, whereas four out of five air leakages were detected with a delay of 2875 observations.

In order to benchmark the studied detection method with regard to the alternative one, the detection of the same simulated air leakages was tested using a moving variance vector that was defined directly from the pressure vector in each simulation round $i$. The window size of the moving variance was 25 , and it moved stepwise along the pressure vector with step size of 25 . Thus, unlike the variance vector $\sigma_{t}^{2}$ of the ARMA +GARCH model, the resulting variance vector had 200 observations where the stable process phase is represented by the 80 observations that were used for the $p_{k}$ quantile definition. To make the detection delays $d_{i}$ comparable, they were multiplied by 25 to transform them back to the same $\mathrm{x}$-axis scale with the Fig. 6, section (a). The cumulative probability curve as illustrated in Fig. 6, section (b), curve (ii), was drawn based on the transformed detection delays.

Fig. 6, section (b), illustrates the cumulative probabilities of the studied detection method with curve (i) and the benchmarked detection method with curve (ii). Their comparison shows that a maximum $56 \%$ of the simulated air leakages were detected faster, i.e. with a shorter delay, when the benchmarked method was used. Methods are equally effective for detecting an air leakage with a delay of 2324 observations. When detection delay exceeds 2324 observations, the studied method becomes more effective.

For the delay of 3000 observations, the studied method failed to detect $16 \%$ of the simulated air leakages whereas the benchmarked method could not detect $37 \%$ of the simulated air leakages. This can be seen from the endpoint of curves (i) and (ii) in the Fig. 5 , section (b).

Fig. 6, section (b), shows that the studied method is slower but more reliable than the benchmarked one, though reducing the type I error rate of the studied method impairs its detection speed and reliability.
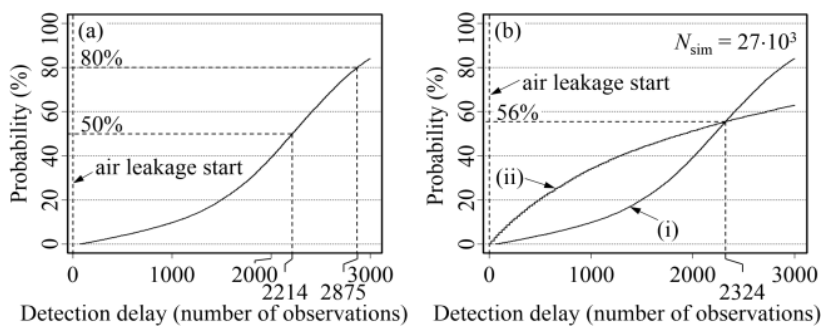

Detection delay (number of observations) Detection delay (number of observations)

Figure 6. (a) Cumulative probability curve of the detection delay $d_{i}$ when detection is based on the variance vector $\sigma_{t}^{2}$ of the combined $\operatorname{ARMA}(4,4)+\operatorname{GARCH}(1,5)$ model. (b) Comparison of the cumulative probability curves: Curve (i) same as in section (a), curve (ii) when detection is based on the moving variance vector that is defined as a moving variance of the simulated pressure vector. 


\section{Conclusions}

An abnormally high variance increase in the critical process variable is often a sign of an incipient failure or other serious disturbance in the process. In this research, the use of the variance vector created using the combined ARMA+GARCH time series model was investigated to detect an abnormal variance increase reliably and with a minimum detection delay. The combined ARMA+GARCH model decomposes a critical variable signal to the standard Gaussian white noise signal and coefficients that determine the AR, MA and the GARCH effects. The variance vector is created based on the coefficients of the GARCH effect. It provides clear visibility to the variance behavior of the critical process variable due to the removed $\mathrm{AR}$ and MA effects that disturb the visibility of the variance behavior. The detection of abnormally high values in the variance vector was carried out by setting a detection limit based on the process phase that is known to be stable and faultless.

A case study was conducted about the critical process variable, i.e. the pressure of the vacuum chamber in the paper machine. Process disturbance, i.e. air leakage in the vacuum chamber, was simulated due to its absence during the pressure measurement. The measured pressure data were used as a basis of the simulation. The simulation results proved that the studied method is somewhat slower but more reliable than the benchmarked one where the controlled variance vector is based on the stepwise moving variance of the critical process variable. The studied method failed to detect $16 \%$ of the simulated air leakages whereas the benchmarked method failed to detect $37 \%$ of the simulated air leakages.

The combined use of the ARMA and GARCH time series models for the Statistical Process Control in industry applications is a fruitful area for further research. When applying these methods in practice, it is beneficial to decompose the data close to their sources using combined ARMA+GARCH time series modelling and to broadcast the decomposition results onward.

\section{References}

T. Bollerslev. Generalized Autoregressive Conditional Heteroskedasticity. Journal of Econometrics, 31(3):307327, 1986. doi:10.1016/0304-4076(86)90063-1

G.E.P. Box and G.M. Jenkins. Time Series Analysis, Forecasting, and Control. Revised Edition, pages 46-84. Oakland, CA: Holden-Day, 1976.

W. Caesarendra, A. Widodo, P.H. Thom, B.-S. Yang and J.D. Setiawan. Combined Probability Approach and Indirect Data-Driven Method for Bearing Degradation Prognostics. IEEE Transactions on Reliability, 60(1):14-20, March, 2011. doi:10.1109/TR.2011.2104716

C. Chen, L.Yu and L. Chen. Structural nonlinear damage detection based on ARMA-GARCH model. Applied Mechanics and Materials, volumes 204-208, pages. 2891-
2896, 2012. doi:10.4028/www.scientific.net/AMM.204208.2891

P.S.P. Cowpertwait and A.V. Metcalfe. Introductory Time Series with $R$. Springer Science+Business Media, page 148. New York, 2009.

S. Du, L. Xi, J. Yu and J. Sun. Online intelligent monitoring and diagnosis of aircraft horizontal stabilizer assembly processes. International Journal of Advanced Manufacturing Technology, 50(1):377-389, 2010. doi:10.1007/s00170-009-2490-0

J. Fan and Q. Yao. Nonlinear Time Series, Nonparametric and Parametric Methods, Springer Science+Business Media, pages 10-13, New York, 2003.

V.K. Jandhyala, S.B. Fotopoulos and D.M. Hawkins. Detection and estimation of abrupt changes in the variability of a process. Computational Statistics \& Data Analysis, 40(1):1-19, 2002. doi:10.1016/S01679473(01)00108-6

F. Li, L. Ye, G. Zhang and G. Meng. Bearing Fault Detection Using Higher-Order Statistics Based ARMA Model. Key Engineering Materials, volume 347, pages 271-276, 2007. doi:10.4028/0-87849-444-8.271

C.M. Meyer and J.F. Zakrajsek. Rocket Engine Failure Detection Using System Identification Techniques. AIAA, SAE, ASME, and ASEE, Joint Propulsion Conference, 26th, Orlando, FL, UNITED STATES, July, 1990.

D.C. Montgomery. Statistical Quality Control A Modern Introduction, Sixth Edition, International Student Version. John Wiley \& Sons, pages 113 and 264, New York, 2009.

H.T. Pham and B.-S. Yang. Estimation and forecasting of machine health condition using ARMA/GARCH model. Mechanical Systems and Signal Processing, 24(2):546558, 2010. doi:10.1016/j.ymssp.2009.08.004

R. Rzadkowski, E. Rokicki, L. Piechowski and R. Szczepanik. Analysis of middle bearing failure in rotor jet engine using tip-timing and tip-clearance techniques. Mechanical Systems and Signal Processing, volumes 76-77, pages 213227, 2016. doi:10.1016/j.ymssp.2016.01.014

R.H. Shumway and D.S. Stoffer. Time Series Analysis and Its Applications: With $R$ Examples, Third Edition, Springer Science+Business Media, pages 83-93,141,286, New York, 2011.

X. Tao, J. Xu, L. Yang and Y. Liu. Bearing fault diagnosis with MSVM based on a GARCH model. Zhendong yu Chongji/Journal of Vibration and Shock, 29(5):11-15, May, 2010.

G. Wang, Z. Luo, X. Qin, Y. Leng and T. Wang. Fault identification and classification of rolling element bearing based on time-varying autoregressive spectrum. Mechanical Systems and Signal Processing, 22(4):934947, 2008. doi:10.1016/j.ymssp.2007.10.008

W. Wang and A.K. Wong. Autoregressive Model-Based Gear Fault Diagnosis. Journal of Vibration and Acoustics, 124(2):172-179, April, 2002. doi:10.1115/1.1456905

L. Zhao, W. Yu and R. Yan. Rolling Bearing Fault Diagnosis Based on CEEMD and Time Series Modeling. Mathematical Problems in Engineering, volume 2014, 2014. Article ID 101867. doi:10.1155/2014/101867 